\title{
DIMENSION AND MEASURE
}

BY

\section{HERBERT FEDERER}

1. Introduction. The relations between topological dimension and Hausdorff measure were discovered by Szpilrajn. (See [SZ], [HW, Chapter VII] ( $\left.{ }^{(}\right)$.) An earlier paper by Pontrjagin and Schnirelman contains related results, though the connection is not explicit. (See [PS].)

According to Szpilrajn, the dimension of a separable metric space $X$ does not exceed $m, \operatorname{dim} X \leqq m$, if and only if $X$ has a homeomorph $Y \subset E_{2 m+1}$ such that the $(m+1)$-dimensional Hausdorff measure of $Y$ equals zero, ${ }^{C_{2 m+1}^{m+1}}(Y)=0$. In the present paper it is shown that the foregoing statement remains true if the Hausdorff measure $\mathcal{S C}_{2 m+1}^{m+1}$ is replaced by the integral geometric Favard measure $\mathcal{F}_{2 m+1}^{m+1}$, or, in fact, by any measure which lies between the Favard measure and the Hausdorff measure, in a sense which is made precise in $\$ 11$. Hence practically all the definitions of $k$-dimensional measure over $n$-space, which may be found in the literature, are equally suitable from the point of view of topological dimension theory.

These results yield new information about the structure of sets of finite Hausdorff measure, which is so intimately tied up. with integral geometric measure (see $[\mathrm{F} 5,9.7]$ ). Suppose $\mathcal{H C}_{n}^{k}(A)<\infty$ and $B$ is the set of all those points of $A$ at which $A$ is not $\left(\mathcal{F C}_{n}^{k}, k\right)$ restricted, which means that $A$ fails to have a $k$-dimensional approximate tangent plane in a suitable sense. Then Szpilrajn's theorem implies that $\operatorname{dim} B \leqq \operatorname{dim} A \leqq k$. However, since $\mathcal{F}_{n}^{\mathbf{k}}(B)=0$, the results of this paper imply that $\operatorname{dim} B \leqq k-1$. This inequality is the best possible.

A formula is proved, which expresses the $k$-dimensional Favard measure of a subset of $n$-space, in terms of the $m$-dimensional Favard measures of its intersections with $(n-k+m)$-dimensional planes, as an integral over the set of all these planes.

To save space, we refer the reader for most of the definitions to [F5]. However, the greater part of this paper may be read without much familiarity with [F5].

2. Definitions. In addition to some of the terms and notations described in $[F 5, \S 2]$, we shall have use for the following:

$p(S)$ is the number (possibily $\infty$ ) of elements of $S$.

If $k<n$ are positive integers, then $p_{n}^{k}$ is the function on $E_{n}$ to $E_{k}$ such that

$$
p_{n}^{k}(x)=\left(x_{1}, x_{2}, \cdots, x_{k}\right) \text { for } x \in E_{n},
$$

Presented to the Society, August 20, 1946; received by the editors January 13, 1947.

(1) Expressions in brackets refer to the bibliography at the end of the paper. 
and $\eta_{n}^{k}$ is the function on $E_{i k}$ to $E_{n}$ such that

$$
\eta_{n}^{k}(y)=\left(y_{1}, y_{2}, \cdots, y_{k}, 0, \cdots, 0\right) \in E_{n} \text { for } y \in E_{k} .
$$

If $s$ and $t$ are positive integers, $x \in E_{s}, y \in E_{t}$, then

$$
(x \circ y)=\left(x_{1}, \cdots, x_{s}, y_{1}, \cdots, y_{t}\right) \in E_{a+t} .
$$

The inverse of the function $f$ will be denoted by inv $f$. [HW].

For the notions of topological dimension theory we refer the reader to

3. The group of distance preserving transformations of $n$-space. Let $M_{n}$ be the set of all distance preserving transformations of $E_{n}$. Thus $f \in M_{n}$ if and only if $f$ is such a function on $E_{n}$ to $E_{n}$ that $|f(x)-f(y)|=|x-y|$ whenever $x \in E_{n}$ and $y \in E_{n}$. We metrize $M_{n}$ by the function $\rho$, which is defined by the formula

$$
\rho(f, g)=|f(\theta)-g(\theta)|+\sup _{|x|=1}|f(x)-f(\theta)-g(x)+g(\theta)|,
$$

where $f \in M_{n}, g \in M_{n}$, and $\theta=(0, \cdots, 0)$ is the origin of $E_{n}$.

Next let

$$
G_{n}=M_{n} \cap \underset{f}{E}[f(\theta)=\theta]
$$

be the set of all orthogonal transformations of $E_{n}$, and associate with each point $x \in E_{n}$ the translation

$$
(x+) \in M_{n}
$$

by means of the relation

$$
(x+)(y)=x+y \text { for } y \in E_{n} .
$$

We recall that the sets $M_{n}$ and $G_{n}$ are groups with respect to the operation, $\therefore$, of superposition, and that each element $f$ of $M_{n}$ has a unique decomposition

$$
f=[(x+): R] \text { with } x \in E_{n}, R \in G_{n} .
$$

If $g$ is another element of $M_{n}$ with the decomposition

$$
g=[(y+): S] \text { with } y \in E_{n}, S \in G_{n},
$$

then

$$
\rho(f, g)=|x-y|+\rho(R, S)
$$

and

$$
(f: g)=\{[(x+R(y))+]:(R: S)\} .
$$

Hence the map of the cartesian product space $\left(E_{n} \times G_{n}\right)$ onto $M_{n}$, which 
associates $[(x+): R]$ with $(x, R)$, is a homeomorphism, though not a homomorphism of the direct product of the groups $E_{n}$ and $G_{n}$ into the group $M_{n}$.

4. The space of $s$-dimensional flat subspaces of $n$-space. Let $\Lambda_{n}^{s}$ be the set of all s-dimensional flat subspaces of $E_{n}$. Let

$$
Z_{n}^{*}=E_{n} \cap \underset{x}{E}\left[x_{i}=0 \text { for } i=1,2, \cdots, n-s\right]
$$

and let $\pi_{n}^{s}$ be the function with domain $M_{n}$ and range $\Lambda_{n}^{s}$ such that

$$
\pi_{n}^{*}(f)=f^{*}\left(Z_{n}^{*}\right) \text { for } f \in M_{n} \text {. }
$$

The maximal sets of constancy of the function $\pi_{n}^{s}$ are the left cosets of the closed subgroup of $M_{n}$ whose elements are those members $f$ of $M_{n}$ for which $f^{*}\left(Z_{n}^{*}\right)=Z_{n}^{*}$. In view of the usual method of assigning a topology to homogeneous spaces, it is natural to give the set $\Lambda_{n}^{*}$ a topology by imposing the condition that

$$
\pi_{n} \text { is an open continuous mapping. }
$$

We next define the function $\lambda_{n}^{;}$on the cartesian product $\left(G_{n} \times E_{n \rightarrow-6}\right)$ to $\Lambda_{n}^{*}$ by the formula

$$
\lambda_{n}^{*}(R, w)=\pi_{n}^{*}\left\{R:\left[\eta_{n}^{n-s}(w)+\right]\right\}
$$

for $R \in G_{n}, w \in E_{n-s .}$

Clearly $\lambda_{n}^{\prime}$ is continuous.

From the relation

$$
(x+)^{*}\left(Z_{n}^{*}\right)=\left[\left(\eta_{n}^{n-s}: p_{n}^{n-s}\right)(x)+\right]^{*}\left(Z_{n}^{*}\right) \text { for } \quad x \in E_{n}
$$

we infer that the range of $\lambda_{n}^{s}$ is $\Lambda_{n}^{s}$.

Now let $\delta$ be the function on $\Lambda_{n}^{s}$ such that $\delta(\mu)$ equals the distance of the set $\mu$ from the origin $\theta=(0, \cdots, 0)$ of $E_{n}$. Clearly $\left(\delta: \pi_{n}^{s}\right)$ is continuous. Therefore $\delta$ is continuous. From this and the relation

$$
\delta\left[\lambda_{n}^{*}(R, w)\right]=|w| \text { for } R \in G_{n}, w \in E_{n-s,}
$$

we infer that:

$A$ set $X \subset \Lambda_{n}^{*}$ is compact if and only if the set [inv $\left.\lambda_{n}^{s}\right]^{*}(X)$ is bounded and closed.

We now associate with each (suitably restricted) function $F$ on $\Lambda_{n}^{s}$ the number $\Psi_{n}^{s}(F)$ by the formula

$$
\Psi_{n}^{*}(F)=\int_{G_{n}} \int_{B_{n \rightarrow}} F\left[\lambda_{n}^{*}(R, w)\right] d \mathcal{L}_{n \rightarrow s} w d \phi_{n} R,
$$

and use the relation 


$$
\{S:(x+)\} *\left[\lambda_{n}^{s}(R, w)\right]=\lambda_{n}^{s}\left\{(S: R),\left[p_{n}^{n-s}: \text { inv } R\right](x)+w\right\},
$$

for $S \in G_{n}, x \in E_{n}, R \in G_{n}, w \in E_{n-s}$, to verify that $\Psi_{n}^{s}$ is invariant under the transformations of $\Lambda_{n}^{s}$ by the elements of $M_{n}$. Furthermore $\left|\Psi_{n}^{*}(F)\right|<\infty$ whenever $F$ is continuous and vanishes outside some compact subset of $\Lambda_{n}^{s}$, and $\Psi_{n}^{s}(F)>0$ whenever $F$ is continuous, nonnegative, and does not vanish everywhere.

It is well known (see [W, p. 45]) that these properties determine $\Psi_{n}^{s}$, up to a constant factor, on the class of all continuous functions on $\Lambda_{n}^{z}$ which vanish outside some compact set, and even on the class of all those functions on $\Lambda_{n}^{s}$ under which each closed set has an analytic counterimage. (See [SS1, pp. $47-50]$.)

5. The integralgeometric measure. If $A$ is an analytic subsét of $E_{n}$, and $k$ is a positive integer less than $n$, then the integralgeometric $k$-dimensional Favard measure of $A$ is given by the formula

$$
\mathcal{f}_{n}^{k}(A)=\beta(n, k)^{-1} \cdot \int_{G_{n}} \int_{E_{k}} p\left[A \cap \lambda_{n}^{n-k}(R, y)\right] d \mathcal{L}_{k} y d \phi_{n} R .
$$

To show that this formula is a consequence of the definition given in $[F 5,2.18]$, we apply the two relations

$$
\begin{gathered}
\mathcal{F}_{n}^{k}(A)=\beta(n, k)^{-1} \cdot \int_{G_{n}} \int_{E_{k}} N\left[\left(p_{n}^{k}: R\right), A, y\right] d \mathcal{L}_{k} y d \phi_{n} R, \\
N\left[\left(p_{n}^{k}: \operatorname{inv} R\right), A, y\right]=p\left[A \cap \lambda_{n}^{n-k}(R, y)\right] \text { for } R \in G_{n}, y \in E_{k} .
\end{gathered}
$$

The first of these formulae was proved in $[\mathrm{F} 5,5.11]$, while the second is verified by the computation:

$$
\begin{aligned}
\lambda_{n}^{n-k}(R, y) & =\left\{R:\left[\eta_{n}^{k}(y)+\right]\right\}^{*}\left(Z_{n}^{n-k}\right) \\
& =R^{*}\left\{E_{n} \cap \underset{x}{E}\left[p_{n}^{k}(x)=y\right]\right\}=E_{n} \cap \underset{z}{E}\left[\left\{p_{n}^{k}: \text { inv } R\right\}(z)=y\right] .
\end{aligned}
$$

This proves our assertion.

We further recall that

$$
\mathcal{F}_{n}^{n}=\mathcal{L}_{n},
$$

and now define $\mathcal{F}_{n}^{0}$ by the formula

$$
\mathscr{f}_{n}^{0}(A)=p(A) \text { for } A \subset E_{n} .
$$

6. Another formula for the invariant integral. Suppose $m<k<n$ are posi. tive integers.

Let

$$
B=\eta_{n}^{n-k+m *}\left(E_{n-k+m}\right)
$$


Since $(n-k+m)+(n-m)-n=n-k$, each flat space $\mu \in \Lambda_{n}^{n-m}$ satisfies exactly one of the three conditions:

(1) $(B \cap \mu)=0$,

(2) $(B \cap \mu) \in \Lambda_{n}^{n-k}$,

(3) the linear dimension of $(B \cap \mu)$ exceeds $(n-k)$.

Let $c$ be the function on $\Lambda_{n}^{n-m}$ such that

$$
\begin{array}{lll}
c(\mu)=0 & \text { if } \quad(1) \text { or }(2) \text { holds, } \\
c(\mu)=1 & \text { if } \quad \text { (3) holds. }
\end{array}
$$

Statement 1. $\Psi_{n}^{n-m i}(c)=0$.

Proof. Let $f$ be the function on $G_{n}$ such that $f(Q)$ is the determinant of the matrix whose columns are

$$
I^{1}, \cdots, I^{n-k+m}, Q^{n-k+m+1}, \cdots, Q^{n},
$$

whenever $Q \in G_{n}$. Here $I$ is the unit matrix in $G_{n}$. Let

$$
S=G_{n} \cap \underset{Q}{E}[f(Q)=0] .
$$

In order to show that

$$
\phi_{n}(S)=0,
$$

we note that $f$ is an analytic function on the analytic Lie group $G_{n}$, that $S$ contains neither component of $G_{n}$, and appeal to the following general proposition:

If the set of zeros of an analytic function on an analytic Lie group intersects a component of the group in a set of positive Haar measure, then it contains that component.

This follows from the fact that all the coefficients of the power series vanish at every point of density of the set of zeros.

Next we see that

$$
c\left[\lambda_{n}^{n-m}(Q, z)\right]=1 \text { implies } Q \in S,
$$

because (3), with $\mu=\lambda_{n}^{n-m}(Q, z)$, implies that the points

$$
I^{1}, \cdots, I^{n-k+m}, Q^{m+1}, \cdots, Q^{n}
$$

fail to span $E_{n}$.

Consequently

$$
\begin{aligned}
\Psi_{n}^{n-m}(c) & =\int_{G_{n}} \int_{E_{m}} c\left[\lambda_{n}^{n-m}(Q, z)\right] d \mathcal{L}_{m} z d \phi_{n} Q \\
& =\int_{S} \int_{E_{m}} c\left[\lambda_{n}^{n-m}(Q, z)\right] d \mathcal{L}_{m} z d \phi_{n} Q=0 .
\end{aligned}
$$

This completes the proof of Statement 1. 
Next we associate with each function $u$ on $\Lambda_{n-k+m}^{n-k}$ the function $u$ on $\Lambda_{n}^{n-m}$ by the formulae

$$
\begin{array}{lll}
\bar{u}(\mu)=u\left[p_{n}^{n-k+m^{*}}(B \cap \mu)\right] & \text { if } & (B \cap \mu) \in \Lambda_{n}^{n-k}, \\
\bar{u}(\mu)=0 & \text { if } & (B \cap \mu) \notin \Lambda_{n}^{n-k} .
\end{array}
$$

Statement 2. If $u$ is such a numerically valued function on $\Lambda_{n-k+m}^{n-k}$ that each closed set has an analytic counterimage under $u$, then

$$
\beta(n-k+m, m) \Psi_{n}^{n-m}(\bar{u})=\beta(n, m) \Psi_{n-k+m}^{n-k}(u) .
$$

Proof. Let $\Omega(u)=\Psi_{n}^{n-m}(\bar{u})$ for each function $u$ of the type just described. If $f \in M_{n-k+m}$, then $g \in M_{n}$, where

$$
g(x)=\left[\eta_{n}^{n-k+m}: f: p_{n}^{n-k+m}\right](x)+x-\left[\eta_{n}^{n-k+m}: p_{n}^{n-k+m}\right](x) \text { for } x \in E_{n},
$$

and we have

$$
\begin{aligned}
\left(p_{n}^{n-k+m}: g\right) & =\left(f: p_{n}^{n-k+m}\right), \quad g^{*}(B)=B, \\
{\left[\left(u: f^{*}\right): p_{n}^{n-k+m^{*}}\right] } & =\left[\left(u: p_{n}^{n-k+m *}\right): g^{*}\right], \\
g^{*}(B \cap \mu) & =B \cap g^{*}(\mu) \quad \text { for } \mu \in \Lambda_{n}^{n-m}, \\
{\left[u: f^{*}\right]\left\{p_{n}^{n-k+m^{*}}(B \cap \mu)\right\} } & =\dot{u}\left\{p_{n}^{n-k+m^{*}}\left[B \cap g^{*}(\mu)\right]\right\} \text { for } \mu \in \Lambda_{n}^{n-m}, \\
\overline{\left(u: f^{*}\right)} & =\left(\bar{u}: g^{*}\right), \quad \Omega\left(u: f^{*}\right)=\Omega(u) .
\end{aligned}
$$

Thus $\Omega$ is invariant under the transformations of $\Lambda_{n-k+m}^{n-k}$ by the elements of $M_{n-k+m}$.

In order to show that $|\Omega(u)|<\infty$ whenever $u$ is continuous and vanishes outside a compact set, and that $\Omega(u)>0$ if $u$ is continuous, nonnegative, and does not vanish everywhere in $\Lambda_{n-k+m}^{n-k}$, it will suffice, by virtue of the uniform structure of $\Lambda_{n-k+m}^{n-k}$, to exhibit a function $u$ with the following properties:

$$
0<\Omega(u)<\infty,
$$

$u$ is nonnegative,

$E[u(\xi) \geqq 1]$ has interior points,

$$
E[u(\xi)>0] \text { has a compact closure. }
$$

To do this, let $\xi$

$$
\begin{aligned}
A & =E_{m} \cap \underset{x}{E}\left[\left|x_{i}\right|<1 \text { for } i=1,2, \cdots, m\right], \\
v(\mu) & =p\left[\eta_{n}^{m *}(A) \cap \mu\right] \quad \text { for } \mu \in \Lambda_{n}^{n-m}, \\
u(\xi) & =p\left[\eta_{n-k+m}^{m}(A) \cap \xi\right] \quad \text { for } \xi \in \Lambda_{n-k+m}^{n-k} .
\end{aligned}
$$


Then $u(\xi) \geqq 1$ for all $\xi$ sufficiently near $Z_{n-k+m}^{n-k}$, and $u(\xi)=0$ whenever the distance of the subset $\xi$ of $E_{n-k+m}$ from the origin of $E_{n-k+m}$ exceeds $m^{1 / 2}$.

Furthermore

$$
\begin{aligned}
\bar{u}(\mu) & =\boldsymbol{p}\left[\eta_{n-k+m}^{m}{ }^{*}(A) \cap p_{n}^{n-k+m *}(B \cap \mu)\right] \\
& =p\left[\eta_{n}^{m^{*}}(A) \cap B \cap \mu\right]=v(\mu)
\end{aligned}
$$

whenever (1) or (2) holds. Hence

$$
\bar{u}(\mu)=v(\mu) \text { whenever } c(\mu)=0,
$$

and we use Statement 1 to infer that

$$
\begin{aligned}
\Omega(u) & =\Psi_{n}^{n-m}(\bar{u})=\Psi_{n}^{n-m}(v)=\beta(n, m) \mathcal{F}_{n}^{m}\left[\eta_{n}^{{ }^{*}}(A)\right] \\
& =\beta(n, m) 2^{m}=\beta(n, m) \mathcal{F}_{n-k+m}^{m}\left[\eta_{n-k+m}^{m}(A)\right] \\
& =[\beta(n, m) / \beta(n-k+m, m)] \Psi_{n-k+m}^{n-k}(u) .
\end{aligned}
$$

Thus $u$ has the required properties, $\Omega$ is a multiple of $\Psi_{n-k+m}^{n-k}$, and the factor equals $[\beta(n, m) / \beta(n-k+m, m)]$.

This completes the proof of Statement 2.

7. The invariance of the Favard measure under an injection.

LеммA. If $m<k<n$ are positive integers, and $X$ is an analytic subset of $E_{n-k+m}$, then

$$
\mathcal{F}_{n}^{m}\left[\eta_{n}^{n-k+m *}(X)\right]=\mathcal{F}_{n-k+m}^{m}(X)
$$

Proof. Let $u$ be the function on $\Lambda_{n-k+m}^{n-k}$ such that

$$
u(\xi)=p(X \cap \xi) \text { for } \xi \in \Lambda_{n-k+m}^{n-k},
$$

and let $v$ be the function on $\Lambda_{n}^{n-m}$ such that

$$
v(\mu)=p\left[\eta_{n}^{n-k+m *}(X) \cap \mu\right] \quad \text { for } \quad \mu \in \Lambda_{n}^{n-m} .
$$

We associate $\bar{u}$ with $u$ as in $\S 6$, and define the function $c$ as in $\S 6$. Then we check that

$$
\bar{u}(\mu)=v(\mu) \text { whenever } c(\mu)=0,
$$

and use Statements 1 and 2 of $\S 6$ to infer that

$$
\begin{aligned}
\beta(n, m) \mathcal{F}_{n}^{m}\left[\eta_{n}^{n-k+m *}(X)\right] & =\Psi_{n}^{n-m}(v)=\Psi_{n}^{n-m}(\bar{u}) \\
& =\beta(n, m) \Psi_{n-k+m}^{n-k}(u) / \beta(n-k+m, m) \\
& =\beta(n, m) \mathcal{F}_{n-k+m}^{m}(X) .
\end{aligned}
$$


The proof is complete.

\section{A new formula for the Favard measure.}

THEOREM. If $n, k, m$ are positive integers, $m<k<n$, and $A$ is an analytic subset of $E_{n}$, then

$$
\mathcal{F}_{n}^{k}(A)=\frac{\beta(n-k+m, m)}{\beta(n, k)} \cdot \int_{G_{n}} \int_{E_{k-m}} \mathcal{F}_{n}^{m}\left[A \cap \lambda_{n}^{n-k+m}(R, w)\right] d \mathcal{L}_{k-m} w d \phi_{n} R .
$$

Proof. Let $g$ be the element of $G_{n}$ such that

$$
g(x)=\left(x_{n-k+m+1}, \cdots, x_{n}, x_{1}, \cdots, x_{n-k+m}\right) \text { for } x \in E_{n},
$$

let

$$
\zeta=\left(g: \eta_{n}^{n-k+m}\right),
$$

and associate with each $S \in G_{n-k+m}$ the function $\bar{S} \in G_{n}$ by the formula

$$
\bar{S}(x)=\left\{p_{n}^{k-m}(x) \circ\left[S: p_{n}^{n-k+m}: \text { inv } g\right](x)\right\} \text { for } x \in E_{n} \text {. }
$$

The remainder of the proof is divided into three parts.

Part 1. If $R \in G_{n}, S \in G_{n-k+m}, w \in E_{k-m}, z \in E_{m}$, then

$$
\lambda_{n}^{n-k}[(R: \bar{S}),(w \circ z)]=\left\{R:\left[\eta_{n}^{k-m}(w)+\right]: \zeta\right\} *\left[\lambda_{n-k+m}^{n-k}(S, z)\right] .
$$

Proof. It is easily verified that

$$
\begin{aligned}
(\bar{S}: \zeta) & =(\zeta: S), \\
{[\zeta:(x+)] } & =\{[\zeta(x)+]: \zeta\} \text { for } x \in E_{n-k+m}, \\
\left(\bar{Q}: \eta_{n}^{k-m}\right) & =\eta_{n} \text { for } Q \in G_{n-k+m}, \\
\left\{\eta_{n}^{k-m}(w)+\left[\zeta: \eta_{n-k+m}^{m}\right](z)\right\} & =\eta_{n}^{k}(w \circ z), \\
\zeta^{*}\left(Z_{n-k+m}^{n-k}\right) & =Z_{n}^{n-k} .
\end{aligned}
$$

Using these relations we infer that

$$
\begin{aligned}
&\left\{R:\left[\eta_{n}^{k-m}(w)+\right]: \zeta: S:\left[\eta_{n-k+m}^{m}(z)+\right]\right\} *\left(Z_{n-k+m}^{n-k}\right) \\
&=\left\{R:\left[\eta_{n}^{k-m}(w)+\right]: \bar{S}: \zeta:\left[\eta_{n-k+m}^{m}(z)+\right]\right\}^{*}\left(Z_{n-k+m}^{n-k}\right) \\
&=\left\{R: \bar{S}:\left[\left(\operatorname{inv} \bar{S}: \eta_{n}^{k-m}\right)(w)+\right]:\left[\left(\zeta: \eta_{n-k+m}\right)(z)+\right]\right\}^{*}\left(Z_{n}^{n-k}\right) \\
&=\left[R: \bar{S}:\left\{\left[\eta_{n}^{k-m}(w)+\left(\zeta: \eta_{n-k+m}^{m}\right)(z)\right]+\right\}\right]^{*}\left(Z_{n}^{n-k}\right) \\
&=\left\{(R: \bar{S}):\left[\eta_{n}^{k}(w \circ z)+\right]\right\}^{*}\left(Z_{n}^{n-k}\right) \\
&=\lambda_{n}^{n-k}[(R: \bar{S}),(w \circ z)] .
\end{aligned}
$$

This proves Part 1.

Part 2. If $R \in G_{n}, w \in E_{k-m}$, then 
544

HERBERT FEDERER

[November

$$
\begin{aligned}
\beta(n-k+m, m) \mathcal{F}_{n}^{m} & {\left[A \cap \lambda_{n}^{n-k+m}(R, w)\right] } \\
& =\int_{G_{n-m+m}} \int_{B_{m}} p\left\{A \cap \lambda_{n}^{n-k}[(R: \bar{S}),(w \circ z)]\right\} d \mathcal{L}_{m} z d \phi_{n-k+m} S .
\end{aligned}
$$

Proof. Let $f=\left\{R:\left[\eta_{m}^{2-m}(w)+\right]: \zeta\right\}$, note that $f$ is a univalent function with domain $E_{n-w+m}$ and range $\lambda_{n}^{n-k+m}(R, w)$, and define

Since

$$
X=\{\operatorname{inv} f\} *\left[A \cap \lambda_{n}^{n-k+m}(R, w)\right] .
$$

$$
f=\left\{R:\left[\eta_{n}^{k-m}(w)+\right]: g: \eta_{n}^{n-k+m}\right\},
$$

we may use the invariance of $\mathcal{F}_{n}^{\mathrm{m}}$ under the transformations of $M_{n}$, together with Lemma 7, to infer that

$$
\mathcal{F}_{n}^{m}\left[A \cap \lambda_{n}^{n-k+m}(R, w)\right]=\mathcal{F}_{n}^{m}\left[f^{*}(X)\right]=\mathcal{F}_{n}^{m}\left[\eta_{n}^{n-k+m^{*}}(X)\right]=\mathcal{F}_{n-k+m}^{m}(X) .
$$

From Part 1 we see that

$$
\begin{aligned}
p\left[X \cap \lambda_{n-k+m}^{n-k}(S, z)\right] & =p\left\{f^{*}\left[X \cap \lambda_{n-k+m}^{n-k}(S, z)\right]\right\} \\
& =p\left\{A \cap \lambda_{n}^{n-k}[(R: S),(w \circ z)]\right\}
\end{aligned}
$$

whenever $S \in G_{n-k+m}, z \in E_{m}$. It follows that

$$
\begin{aligned}
\beta(n-k+m, m) & \mathcal{F}_{n-k+m}^{m}(X) \\
& =\int_{G_{n-k+m}} \int_{B_{m}} p\left\{A \cap \lambda_{n}^{n-k}[(R: \bar{S}),(w \circ z)]\right\} d \mathcal{L}_{m} z d \phi_{n-k+m} S .
\end{aligned}
$$

This proves Part 2.

Part 3.

$$
\begin{aligned}
& \beta(n, k) \mathcal{F}_{n}^{k}(A)=\beta(n-k+m, m) \\
& \cdot \int_{G_{n}} \int_{B_{k-\infty}} \mathcal{F}_{n}^{m}\left[A \cap \lambda_{n}^{n-k+m}(R, w)\right] d \mathcal{L}_{k-m} w d \phi_{n} R .
\end{aligned}
$$

Proof. Using 5 and Part 2, we compute

$$
\begin{aligned}
& \beta(n, k) \mathcal{F}_{n}^{k}(A)=\int_{G_{n}} \int_{B_{k}} p\left[A \cap \lambda_{n}^{n-k}(R, y)\right] d \mathcal{L}_{k} y d \phi_{n} R \\
&=\int_{G_{n-k+m}} \int_{G_{n}} \int_{B_{m}} \int_{B_{k-m}} p\left\{A \cap \lambda_{n}^{n-k}[(R: \bar{S}),(w \circ z)]\right\} d \mathcal{L}_{n-m} w d \mathcal{C}_{m} z d \phi_{n} R d \phi_{n-k+m} S \\
&=\int_{G_{n}} \int_{E_{k-m}} \beta(n-k+m, m) \mathcal{F}_{n}^{m}\left[A \cap \lambda_{n}^{n-k+m}(R, w)\right] d \mathcal{L}_{k-m} w d \phi_{n} R .
\end{aligned}
$$


The proof is complete.

9. Dimension and Favard measure.

THEOREM. If $s$ and $n$ are integers, $n>0,0 \leqq s \leqq n$, and $\mathcal{F}_{n}^{*}(A)=0$, then $\operatorname{dim} A \leqq s-1$.

Proof. We fix $n$ and use induction in $s$.

Let $S$ be the set of all integers $s$ for which there is a set $A$ with $\mathcal{F}_{n}^{a}(A)=0$ and $\operatorname{dim} A \geqq s$.

Assume that $S \neq 0$, and let $k$ be the least integer in $S$. The proof that this assumption leads to a contradiction is divided into three parts.

Part 1. $k>0$.

Proof. Otherwise $k=0$ and there is a set $A$ for which

$$
\operatorname{dim} A \geqq 0, \quad \mathscr{F}_{n}^{0}(A)=0 .
$$

Then $p(A)=0, A=0, \operatorname{dim} A=-1$.

This contradiction proves Part 1.

Part 2. $k=n$.

Proof. Otherwise Part 1 implies $0<k<n$, and, since every set is contained in an analytic set of equal Favard measure, there is an analytic set $A$ for which

$$
\operatorname{dim} A \geqq k, \quad \mathcal{F}_{n}^{k}(A)=0 .
$$

The theorem of $\S 8$ implies that

$$
\int_{G_{n}} \int_{E_{1}} \mathcal{F}_{n}^{k-1}\left[A \cap \lambda_{n}^{n-1}(R, w)\right] d \mathcal{L}_{1} w d \phi_{n} R=0 .
$$

Letting $U$ be the set of all $R \in G_{n}$ for which

$$
\int_{E_{1}} \mathcal{F}_{n}^{k-1}\left[A \cap \lambda_{n}^{n-1}(R, w)\right] d \mathcal{L}_{1} w=0,
$$

we infer that

$$
\phi_{n}\left(G_{n}-U\right)=0 .
$$

Next choose such members ${ }^{1} Q,{ }^{2} Q, \cdots,{ }^{n} Q$ of $G_{n}$ that

$$
(Q)^{1}=I^{i} \text { for } i=1,2, \cdots, n \text {. }
$$

Here $I$ is the unit matrix of $G_{n}$.

Since

$$
\phi_{n}\left\{G_{n}-\underset{R}{E}[(R: Q) \in U]\right\}=0 \text { for } i=1,2, \cdots, n,
$$

we conclude that 


$$
\left(R:{ }^{i} Q\right) \in U \text { for } i=1,2, \cdots, n
$$

for $\phi_{n}$ almost all $R \in G_{n}$.

It is easily seen that

$$
\lambda_{n}^{n-1}(V, w)=E_{n} \cap \underset{x}{E}\left[x \cdot V^{1}=w\right]
$$

whenever $V \in G_{n}, w \in E_{1}$.

We now pick $R \in G_{n}$ such that

$$
\left(R:{ }^{i} Q\right) \in U \text { for } i=1,2, \cdots, n
$$

and use the relation $\left(R:^{i} Q\right)^{1}=R^{i}$ to infer that

$$
\mathcal{F}_{n}^{k-1}\left\{A \cap \underset{x}{E}\left[x \cdot R^{i}=w\right]\right\}=0
$$

for $\mathcal{L}_{1}$ almost all $w$ in $E_{1}$, if $i$ is an integer between 1 and $n$.

Therefore the $(n-1)$-planes of the type

$$
E_{n} \cap \underset{x}{E}\left[x \cdot R^{i}=w\right],
$$

which intersect $A$ in a set of (k-1)-dimensional Favard measure zero, contain all the faces of arbitrarily small cubical neighborhoods of each point of $E_{n}$. Hence the boundary of each of these cubical neighborhoods intersects $A$ in a set of $(k-1)$-dimensional Favard measure zero, whose topological dimension does not exceed $(k-2)$, because $k$ is the least integer in $S$.

It follows that

$$
\operatorname{dim} A \leqq k-1 .
$$

This completes the proof of Part 2.

Part 3. $k \neq n$.

Proof. Otherwise there is a set $A$ for which

$$
\operatorname{dim} A=n, \quad \mathcal{L}_{n}(A)=0 .
$$

Hence $A$ both contains, and does not contain, a nonvacuous open subset. This completes the proof.

REMARK. An alternate proof of Part 3 runs somewhat like the proof of Part 2. Fubini's theorem may be used to construct an appropriate grating of $(n-1)$-planes.

10. Application to sets of finite Hausdorff measure. Suppose $k<n$ are positive integers, $A$ is an $\mathcal{F C}_{n}^{k}$ measurable subset of $E_{n}$, and $\mathcal{F C}_{n}^{k}(A)<\infty$. Then

$$
A=A_{1} \cup A_{2},
$$

where $A_{1}$ is the set of all points of $A$ at which $A$ is $\left(\mathcal{F C}_{n}^{k}, k\right)$ restricted, and $A_{2}=A-A_{1}$. 
As in $[\mathrm{F} 5,9.7]$ we infer that $\mathcal{F}_{n}^{k}\left(A_{1}\right)=\mathcal{F C}_{n}^{k}\left(A_{1}\right), \mathcal{F}_{n}^{k}\left(A_{2}\right)=0$.

Therefore $\mathcal{F}_{n}^{k+1}\left(A_{1}\right)=0$, and Theorem 9 assures us that

$$
\operatorname{dim} A_{1} \leqq k, \quad \operatorname{dim} A_{2} \leqq k-1 .
$$

These inequalities are the best possible, for each pair $(k, n)$.

11. The general relation between dimension and measure. Suppose $\psi$ is any double sequence of measures with the following properties:

(i) If $k$ and $n$ are integers, $n>0,0 \leqq k \leqq n$, then $\psi_{n}^{k}$ is a measure over $E_{n}$.

(ii) If $\mathfrak{F C}_{n}^{k}(A)=0$, then $\psi_{n}^{k}(A)=0$.

(iii) If $\psi_{n}^{k}(A)=0$, then $\mathcal{F}_{n}^{k}(A)=0$.

Here $\mathfrak{F C}_{n}^{0}(A)=\mathcal{F}_{n}^{0}(A)=p(A)$ for $A \subset E_{n}$.

Then we may use one half of Szpilrajn's theorem, namely [HW, Theorem VII 4], together with the theorem of $\$ 9$ of this paper, to conclude:

If $X$ is a separable metric space, then

$$
\operatorname{dim} X \leqq m
$$

if and only if $X$ has a homeomorph $Y$ such that

$$
Y \subset E_{2 m+1}, \quad \psi_{2 m+1}^{m+1}(Y)=0 .
$$

The conditions imposed on $\psi$ are satisfied by most definitions of measure which can be found in the literature. In particular, we may take $\psi_{n}^{k}$ to be any one of the seven measures $S_{n}^{k}, \mathcal{F}_{n}^{k}, \mathcal{C}_{n}^{k}, \Phi_{n}^{k}, \Gamma_{n}^{k}, G_{n}^{k}, \mathcal{F}_{n}^{k}$ discussed in $[F 5,2.18]$.

This shows that all these measures are equally suitable from the point of view of dimension theory.

\section{BIBLIOGRAPHY}

The following list is a selection from the bibliography of [F5].

H. FEDERER

[F4] Coincidence functions and their integrals, Trans. Amer. Math. Soc. vol. 59 (1946) pp. 441-466.

[F5] The $(\phi, k)$ rectifiable subsets of $n$-space, Trans. Amer. Math. Soc. vol. 62 (1947) pp. 114-192.

W. HuREWicz and H. Wallman

[HW] Dimension theory, Princeton Mathematical Series, Princeton University Press, 1941.

L. Pontrjagin and L. Schnirelman

[PS] Sur une propriêté metrique de la dimension, Ann. of Math. vol. 33 (1932), pp. 156-162.

S. SAKS

[SS1] Theory of the integral, Warsaw, 1937.

E. SzPILRAjN

[SZ] La dimension et la mesure, Fund. Math. vol. 28 (19.37) pp. 81-89.

A. WEIL

[W] L'intégration dans les groupes topologiques et ses applications, Actualités Scientifiques et Industrielles, vol. 869, Hermann, Paris, 1938.

BROWN UNIVERSITY,

Providence, R. I. 